\title{
Acute Myeloid Leukemia with Germline CEBPA Mutation
}

National Cancer Institute

\section{Source}

National Cancer Institute. Acute Myeloid Leukemia with Germline CEBPA Mutation. NCI

Thesaurus. Code C151898.

Familial acute myeloid leukemia (AML) syndrome associated with biallelic CEBPA

mutations. Patients typically present with AML as children or young adults. The familial form of AML with germline CEBPA mutation has morphological and immunophenotypic features similar to those of sporadic AML with CEBPA mutations. Overall, it has a favorable prognosis. (WHO 2017) 